\title{
European Hernia Society classification of parastomal hernias
}

\author{
M. Śmietański • M. Szczepkowski • J. A. Alexandre • D. Berger • \\ K. Bury $\cdot$ J. Conze $\cdot$ B. Hansson $\cdot$ A. Janes $\cdot$ M. Miserez $\cdot$ V. Mandala \\ A. Montgomery $\cdot$ S. Morales Conde $\cdot$ F. Muysoms
}

Received: 7 May 2013/Accepted: 14 September 2013/Published online: 1 October 2013

(C) The Author(s) 2013. This article is published with open access at Springerlink.com

\begin{abstract}
Purpose A classification of parastomal hernias ( $\mathrm{PH})$ is needed to compare different populations described in various trials and cohort studies, complete the previous inguinal and ventral hernia classifications of the European Hernia Society (EHS) and will be integrated into the EuraHS database (European Registry of Abdominal Wall Hernias).

Methods Several members of the EHS board and invited experts gathered for 2 days to discuss the development of an EHS classification of PH. The discussions were based on a literature review and critical appraisal of existing classifications.

Results The classification proposal is based on the $\mathrm{PH}$ defect size (small is $\leq 5 \mathrm{~cm}$ ) and the presence of a concomitant incisional hernia (cIH). Four types were defined: Type I, small PH without cIH; Type II, small PH with cIH; Type III, large PH without cIH; and Type IV, large PH
\end{abstract}

M. Śmietański

Department of General and Vascular Surgery,

Hospital in Wejherowo, Wejherowo, Poland

M. Śmietański ( $\square)$

EHS Secretary Scientific Research Office, ul. Borsucza 12b, 80-175 Gdańsk, Poland

e-mail: smietana@gumed.edu.pl

M. Szczepkowski

Department of Rehabilitation, Pilsudski University of Physical

Education, Warsaw, Poland

M. Szczepkowski

Clinical Department of General and Colorectal Surgery,

Bielanski Hospital, Warsaw, Poland

\section{J. A. Alexandre}

Department of General Surgery, Université Paris 6, Paris, France with cIH. In addition, the classification grid includes details about whether the hernia recurs after a previous $\mathrm{PH}$ repair or whether it is a primary $\mathrm{PH}$. Clinical validation is needed in the future to assess if the classification allows us to differentiate the treatment strategy and if the classification impacts outcome in these different subgroups.

Conclusion A classification of PH divided into subgroups according to size and $\mathrm{cIH}$ was formulated with the aim of improving the ability to compare different studies and their results.

Keywords Parastomal $\cdot$ Hernia $\cdot$ Classification

\section{Introduction}

Recently, the European Hernia Society (EHS) has systematised and introduced classifications of inguinal and ventral (primary and incisional) hernias [1, 2]. Both

D. Berger

Department of Surgery, Stadtklinik Baden-Baden,

Baden-Baden, Germany

K. Bury

Department of Cardiac and Vascular Surgery, Medical

University of Gdansk, Gdansk, Poland

J. Conze

Department of Surgery, University Clinic Aachen,

Aachen, Germany

B. Hansson

Department of Surgery, Canisius Wilhelmina Hospital, Nijmegen, Netherlands

\section{A. Janes}

Department of Surgery, Ortopedteknik Sundsvalls sjukhus, Sundsvalls, Sweden 
classifications are based on the stipulation that a good classification must be simple; these classifications should mention the most appropriate and practicable criteria. The classification of ventral hernia is used in another EHS project-the European Registry of Abdominal Wall Hernias (EuraHS) [3]. This database covers the registry of parastomal hernias $(\mathrm{PH})$; the founders of this project stated that a classification of $\mathrm{PH}$ is still missing. Previously published classifications of $\mathrm{PH}$ are not practicable and have not been used in any publication other than the original publication. Most of the previous proposals did not mention concomitant incisional hernias $(\mathrm{cIH})$ and included subgroups that did not qualify as hernias, according to the definition of abdominal wall hernias as proposed by the EHS [4-8]. During the EHS board meeting in Madonna di Campilio (February 2011), the governors agreed to organise a consensus conference. The aim was to introduce an EHS classification for PH based on the same template and rules of previously published EHS classifications for inguinal and ventral hernias to improve the ability to compare different studies and their results.

\section{Materials and methods}

The conference was held on behalf of the Secretary for Scientific Research in Torun, Poland, on April 20-21, 2012. Several members of the EHS board and invited experts on $\mathrm{PH}$ met for 2 days to discuss currently existing classifications and the possibility of developing a new or modified system [8] (Fig. 1). Participants were chosen based on a literature review of publications on $\mathrm{PH}$ from leading recognised centres; the participants were approved by the EHS board (Fig. 2).

The participants agreed on the general rules of classification before their meeting. According to the ontology, unambiguous interpretation of the classes and strict

\section{Miserez}

Department of Abdominal Surgery, Universitair Ziekenhuis Leuven, Louvain, Belgium

\section{Mandala}

Department of General and Emergency Surgery, Villa SoWa, CTO Hospital, Palermo, Italy

\section{A. Montgomery}

Department of Surgery, Malmo University Hospital,

Malmo, Sweden

\section{S. Morales Conde}

Department of Surgery, University of Sevilla, Seville, Spain

F. Muysoms

Department of Surgery, AZ Maria Middelares, Ghent, Belgium

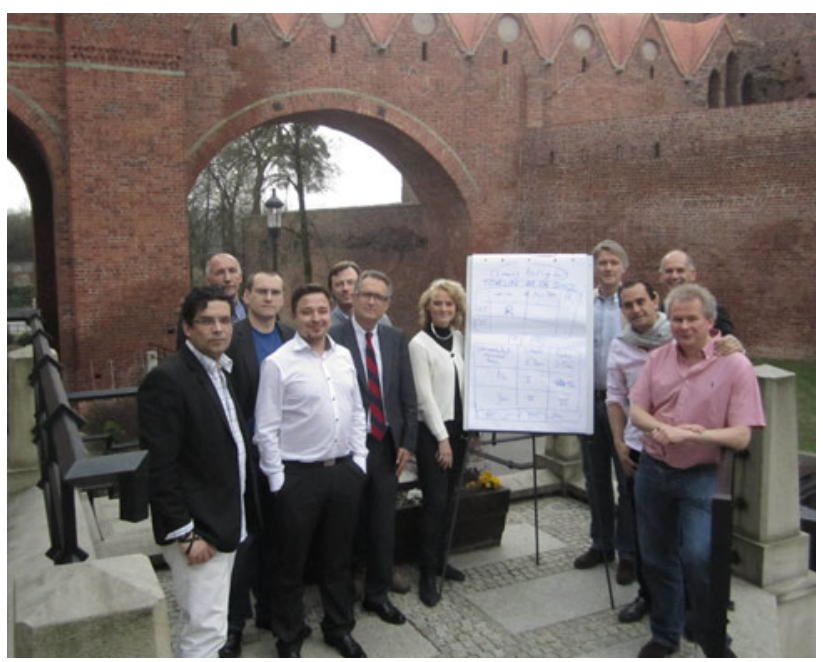

Fig. 1 The EHS classification for parastomal hernias is a proposal formulated during a consensus meeting in Torun, Poland, on April 20-21, 2012

hierarchical subclass relationship were needed. To make this classification useful, we decided that the level of granulation (measurements needed to classify) should be low, and natural language should be used. By mentioning the "natural language" abandonment of abstract abbreviations, we targeted sharp classification of meaning of terms and the challenge of pluralism of the cultures. These rules are usually useful in the methodology of taxonomy; they are used to validate various classifications.

Creating a successful classification in the medical community implies that surgeons must be convinced of the value of the proposal; additionally, they must accept the information required and uniformly complete the data sets. It is important to create a proposal that uses clearly defined criteria from which we can anticipate a positive effect of the treatment strategy and prognosis.

Currently existing classifications were reviewed with respect to the number of different subgroups, relevance, missing subgroups, accuracy and simplicity.

\section{Results}

Currently existing classifications

Previously published classifications on $\mathrm{PH}$ are based on three different examination methods: physical examination, intraoperative findings or radiological description (CT scans or US). According to the type of hernia and hernia content, we isolated four to five subgroups of hernias. These subgroups describe various pathological findings of the stoma: from the real hernias (with the presence of the hernia sac and its content) to the prolapse of the stoma or 

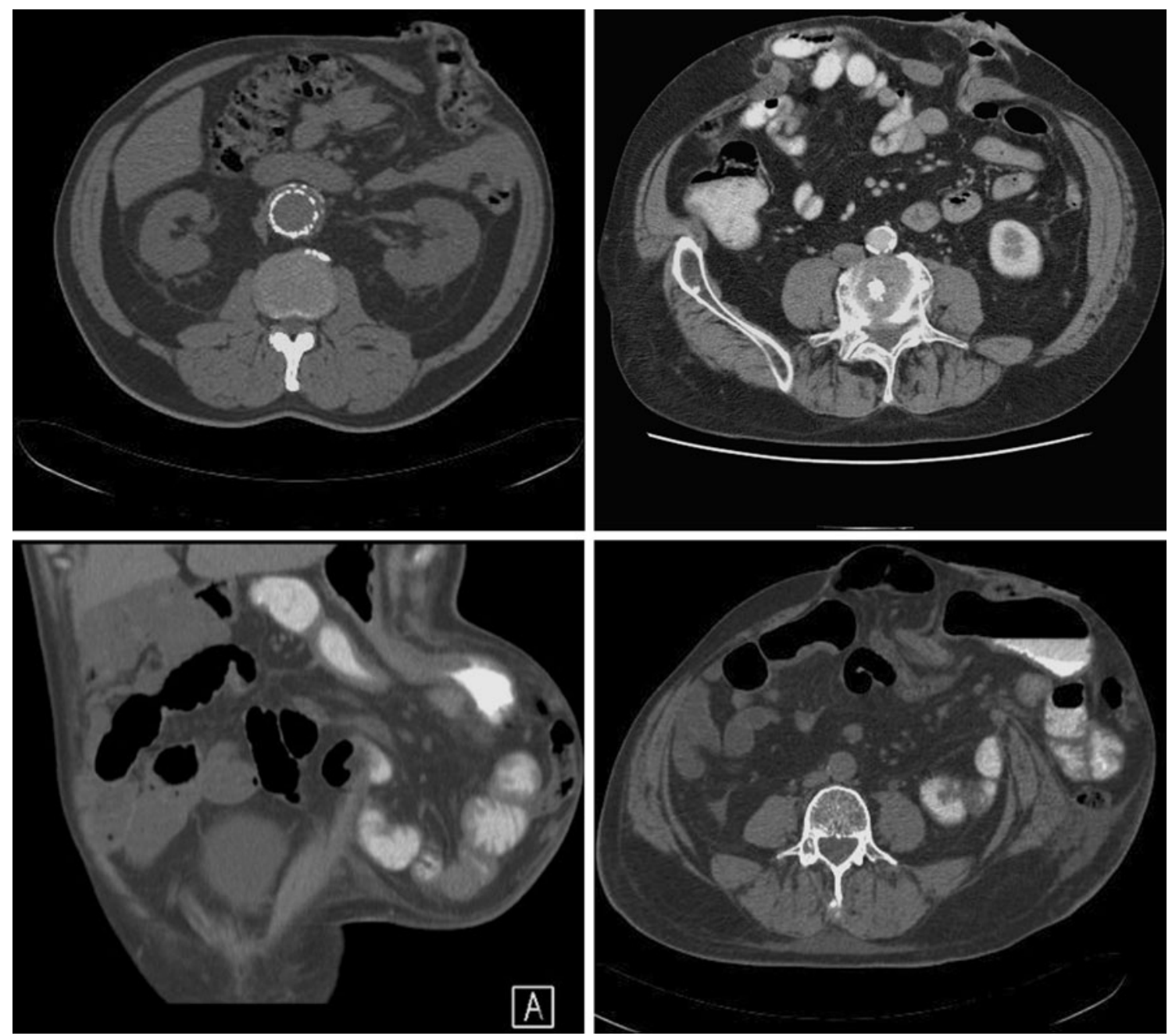

Fig. 2 Examples of the different subgroups of parastomal hernias as defined by the EHS classification

Table 1 Description of previous parastomal hernia classification proposals

\begin{tabular}{lllll}
\hline Author (year) & Classification type & Classification based on & Number of subclasses & Clinical validation \\
\hline Devlin [5] & Intraoperative & Intraoperative findings & 4 & Yes \\
Rubin [4] & Intraoperative & Intraoperative findings & 4 & No \\
Moreno-Matias [6] & Radiological & CT & 5 & Yes \\
Gil, Szczepkowski [Bielanski Hospital] [8] & Clinical & Physical examination & 4 & Yes
\end{tabular}

stoma loop protrusion under the skin [4-7]. Only the classification by Gil and Szczepkowski includes coexisting midline incisional hernias in the classification [8]. Table 1 summarises all published classifications. Table 2 summarises the subgroup definitions of different classifications.

Existing classifications are of low clinical value, except for the one described by Gil and Szczepkowski [8]. They have not been used in any clinical trial or cohort group description; it seems that the subgroup allocation of patients will not influence the treatment options or the prognosis. The classifications proposed by Devlin and
Rubin include subgroups that do not fulfil the definition of hernia $[4,5]$. The allocation of a patient to the appropriate type or subgroup is impossible on clinical examination alone, except for the Gil and Szczepkowski classification. Indications for treatment were often taken into consideration while creating classifications. The classification by Moreno-Matias was evaluated according to the presence of symptoms reported by the patient [6]. In Type 0 , over 70 percent of hernias were asymptomatic, whereas in Type III, all of the hernias manifested clinical symptoms [6]. A publication by Seo has confirmed those findings [7]. The 
Table 2 Subgroups of parastomal hernias in various classification proposals

\begin{tabular}{ll}
\hline Devlin [5] & Rubin et al. [4] \\
Type I: interstitial hernia & Type I: true parastomal hernia \\
Type II: subcutaneous hernia & Ia: interstitial \\
Type III: intrastomal hernia & Ib: subcutaneous \\
Type IV: peristomal hernia (stoma prolapse) & Type II: intrastomal hernia \\
& Type III: subcutaneous prolapse \\
& Type IV: pseudohernia (connected with flank insufficiency or denervation) \\
Moreno-Matias [6] & Gil and Szcepkowski [8] \\
Type 0: Peritoneum follows the wall of the bowel & Type I: isolated small parastomal hernia \\
forming the stoma, with no formation of a sac & Type II: small parastomal hernia with coexisting midline incisional hernia \\
Type Ia: Bowel forming the colostomy with a sac $<5 \mathrm{~cm}$ & (without any significant front abdominal wall deformity) \\
Type Ib: Bowel forming the colostomy with a sac $>5 \mathrm{~cm}$ & Type III: isolated large parastomal hernia (with significant front abdominal wall \\
Type II: Sac containing omentum & deformity) \\
Type III: Intestinal & Type IV: large parastomal hernia with coexisting midline incisional hernia (with \\
loop other than the bowel forming the stoma & significant front abdominal wall deformity)
\end{tabular}

classification proposed by Gil et al. described the clinical symptoms in different subclasses. The most frequent indications for treatment in Type I were strangulation and ileus. In Type II, local complications dominated among the patients. In Types III and IV, the size of the hernia and the deformity of the front abdominal wall or flank were considered as indications [8]. Nevertheless, in this classification, the cut-offs of subclasses were not precisely defined ("small" vs. "large"), which can lead to mistakes and can make comparative studies impossible.

A pure classification does not include the type of operative technique used. However, it seems that the complexity of the operation will increase with the described subgroups.

\section{Purpose of the classification}

The primary purpose of the work was to improve the ability to compare different studies and their results. The secondary purpose was to use the classification in various databases, such as the EuraHS database, to collect the data. We believe that if the proposed classification is widely accepted and used, the possibility of developing evidencebased therapeutic guidelines will increase in the future. Validation of the classification is though needed.

Format of the classification

In 2007, the EHS published the classification of inguinal hernias; in 2009, based on a similar template, a ventral hernia classification was proposed [1, 2]. We agreed that $\mathrm{PH}$ classification should have a similar format and that a grid format should be proposed, even if it restricts the number of variables that can be used for the classification.
Definition of a parastomal hernia

Following the EHS definition of ventral hernia (Any abdominal wall gap with or without a bulge in the area of a postoperative scar perceptible or palpable by clinical examination or imaging [1]), $\mathrm{PH}$ is an abnormal protrusion of the contents of the abdominal cavity through the abdominal wall defect created during placement of a colostomy, ileostomy or ileal conduit stoma [3]. It should be distinguished from local stoma problems without a hernia sac, such as a mucosal prolapse or a Siphon loop, which is a subcutaneous folding of the excess bowel length at the stoma.

Variables for classification

When proposing a classification, it is important to determine the most suitable variables that should be included. We have considered many potential criteria that influence the symptomatology of the hernia, the choice of treatment and treatment prognosis (Table 3). It is impossible to consider all of these variables because it would make the proposed classification too extensive and complicated. Therefore, a consensus decision on the exclusion or inclusion of variables was made.

\section{Discussion}

Choice of variables

The task of developing a good classification of $\mathrm{PH}$ is similar to the classification of incisional hernias. There is great variability in the morphology of $\mathrm{PH}$; additionally, we agreed that coexistence of another incisional hernia in the 
Table 3 Variables discussed to be included in the final classification

$\begin{array}{lll}\text { Variable proposed for classification } & \text { Included } & \text { Excluded }\end{array}$ (description)

Patients' medical history (cancer, bowel inflammatory disease, etc.)

Risk factors

Type of stomy (colostomy, ileostomy, urostomy, ileal conduit)

Localisation (according to the EHS ventral hernia classification)

Hernia symptoms (ileus, bowel obstruction, pain, etc.)

Local stoma problems (fistula, skin lesion or necrosis, etc.)

Defect size (largest diameter)

$\mathrm{X}$

Size of the hernia sac

Hernia content (stoma loop, other bowel, omentum, etc.)

Coexisting incisional hernia (previous scar in $\mathrm{X}$ the middle line)

Distance between parastomal hernia and midline (ev. midline hernia border)

Recurrence

$\mathrm{X}$

previous scar will change treatment and may influence the outcome. There was consensus that the presence of a cIH and the size of the $\mathrm{PH}$ defect play a crucial role in the proposed treatment. Therefore, we chose to base the new classification upon the Szczepkowski classification [8]. We added the cut-off value of $5 \mathrm{~cm}$ to distinguish a small $\mathrm{PH}$ from a large PH. The size of the hernia defect is defined as the largest diameter of the hernia orifice in any direction, the width, the length or a diagonal measurement. After careful discussion cut-off value was stated as $5 \mathrm{~cm}$. Authors discussed the material from study conducted by Hansson et al [9]. It was mentioned that almost half (41\%) of patients has $\mathrm{cIH}$, and in almost $50 \%$ the size of parastomal defect was larger than $5 \mathrm{~cm}$. This leads to the conclusion that choosing this variables as cut-off value will divide the patients population in four almost equal subgroups, what can help in future treatment strategy choice. In addition, the descriptions of the deformation of the front abdominal wall have been removed from the classification because they are difficult to define and might lead to decreased reproducibility (Table 2). To make the new classification similar to previously published inguinal and incisional hernia classifications, a separate box was added to the grid describing primary or recurrent hernia (as in other classifications, $\mathrm{P}$ indicates primary and $\mathrm{R}$ indicates recurrent).

Other discussed variables and risk factors would make the classification too complex. Although they could be important for the treatment choice and prognosis, all of them are included in the EuraHS database, so that they could be analysed in the future if more clinical cases are reported in the registry. We also believe that some variables such as medical history and risk factors are of minor importance for the choice of surgical approach and technique. These variables can be important for the general clinical outcome of the patient (e.g., an existing malignancy). In most circumstances, local stoma problems, localisation, symptoms of the hernia, size of the sac and its content do not influence the operative technique itself. These types of variables were also excluded from other classifications.

Subclasses of classification were defined as follows (Fig. 2):

Type I: $\mathrm{PH} \leq 5 \mathrm{~cm}$ without $\mathrm{cIH}$.

Type II: $\mathrm{PH} \leq 5 \mathrm{~cm}$ with $\mathrm{cIH}$.

Type III: $\mathrm{PH}>5 \mathrm{~cm}$ without $\mathrm{cIH}$.

Type IV: $\mathrm{PH}>5 \mathrm{~cm}$ with $\mathrm{cIH}$.

$\mathrm{P}$ : primary $\mathrm{PH}$.

$\mathrm{R}$ : recurrence after previous $\mathrm{PH}$ treatment.

\section{Classification table}

In Table 4, we propose a format for the classification of PH.

How to use a classification

As in previously published inguinal and ventral hernia and parastomal classifications, the measurements of the hernia orifice and $\mathrm{cIH}$ are performed intraoperatively. The measure of the hernia orifice should follow the general roles of previously published EHS classification of incisional hernias (aggregate length and width of all defects on previous scar [1], but the size of the parastomal defect separately).

Table 4 EHS grid for classification of parastomal hernias

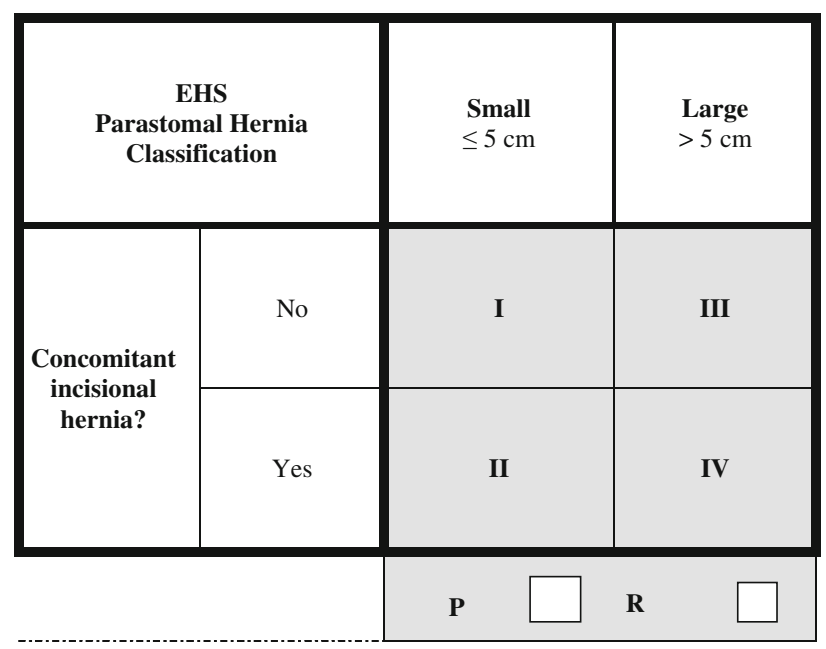


We agree that although CT scans could be performed preoperatively and could help determine the subgroup of the defect, intraoperative measurement is the recommended procedure.

\section{Conclusions}

The aim of this project was achieved. It was possible to create a group of experts on behalf of the EHS to propose a new classification of $\mathrm{PH}$. We believe that due to its simplicity and the sharp edges of the subgroup definition, it will help surgeons to formulate a precise description of the pathology. The proposed classification is also the missing link in the EHS classifications of abdominal wall hernias. Although many variables were not included in the classification, we believe that, together with the data collected in the registers (including EuraHS), this work will bring us more information about PH treatment in the future. Similar to the inguinal and ventral hernia classification, we believe that the proposed classification must be tested and validated in clinical practice. The validation process might provide new information to allow us to consider future modifications of the classifications.

Open Access This article is distributed under the terms of the Creative Commons Attribution Noncommercial License which permits any noncommercial use, distribution, and reproduction in any medium, provided the original author(s) and the source are credited.

\section{References}

1. Muysoms F, Miserez M, Berrevoet F et al (2009) Classification of primary and incisional abdominal wall hernias. Hernia 13:407-414

2. Miserez M, Alexandre JH, Campanelli G (2007) The european hernia society groin hernia classification: simple and easy to remember. Hernia 11:113-116

3. Muysoms F, Campanelli G, Champault GG (2012) EuraHS: the development of an international online platform for registration and outcome measurement of ventral abdominal wall hernia repair. Hernia 16:239-250

4. Rubin MS, Schoetz DJ, Matthews JB (1994) Parastomal hernia. Is stoma relocation superior to fascial repair? Arch Surg 129:413-418

5. Devlin HB, Kingsnorth A (1998) Management of abdominal hernias. London, Hodder Arnold Publishers, pp 177-178

6. Moreno-Matias J et al (2009) The prevalence of parastomal hernia after formation of an end colostomy. A new clinico-radiological classification. J Colorectal Dis 11:173-177

7. Seo HS, Kim HJ, Sy Oh, Lee JH, Suh KW (2011) Computed tomography classification for parastomal hernia. J Korean Surg Soc 81:111-114

8. Gil G, Szczepkowski MS (2011) A new classification of parastomal hernias-from the experience at Bielański Hospital in Warsaw. Pol J Surg 83:430-437

9. Hansson BME, Morales-Conde S, Mussac T et al (2013) The laparoscopic modified Sugarbaker technique is safe and has a low recurrence rate: a multicenter cohort study. Surg Endosc 27:494-500 